\title{
Inhibitory effect of rice bran-derived crude glycosphingolipid on colon preneoplastic biomarker lesions induced by azoxymethane in male F344 rats
}

\author{
NAO SUNAGAWA ${ }^{1}$, MORIHIKO INAMINE $^{2}$, TAKAMITSU MORIOKA ${ }^{1}$, ITARU CHIBA $^{1}$, \\ NANAE MORITA $^{1}$, YOICHI AOKI ${ }^{2}$, MASUMI SUZUI ${ }^{3}$ and NAOKI YOSHIMI ${ }^{1}$
}

Departments of ${ }^{1}$ Tumor Pathology, and ${ }^{2}$ Obstetrics and Gynecology, University of the Ryukyus Faculty of Medicine, 207 Uehara, Nishihara-cho, Okinawa 903-0215; ${ }^{3}$ Medical Therapeutics and Molecular Therapeutics, Gifu Pharmaceutical University, 5-6-1 Mitadouhigasi, Gifu-city, Gifu 502-8585, Japan

Received September 2, 2008; Accepted October 9, 2008

DOI: 10.3892/mmr_00000060

\begin{abstract}
The aim of the present study was to examine whether crude glycosphingolipid (cGSL) has short-term chemopreventive effects on the preneoplastic biomarker lesions involved in carcinogen-induced rat colon carcinogenesis. We also examined whether cGSL affects cell proliferation and apoptosis in these lesions. The crude preparation was obtained by the simple ethanol extraction method. Five-week-old male F344 rats were divided into 6 groups. Rats in groups 1-4 were given subcutaneous injections of azoxymethane (AOM) (20 mg/kg body weight) once a week for 2 weeks. Starting 1 week before the first injection of AOM, the rats in groups 2, 3 and 4 were fed a diet containing 250,1,000 and 3,000 ppm cGSL, respectively, for 5 weeks. The experiment was terminated 5 weeks after the start date, and the number of aberrant crypt foci (ACF) and mucin-depleted foci (MDF) was counted. Dietary cGSL significantly inhibited the induction of ACF (group 3, $\mathrm{P}<0.01$; group 4, $\mathrm{P}<0.05$ ) and $\mathrm{MDF}$ (groups 2 and 3, $\mathrm{P}<0.001$; group 4, $\mathrm{P}<0.05)$ as compared to group 1 treated with $\mathrm{AOM}$ alone. In groups 3 and 4 , proliferating cell nuclear antigen-positive indices of epithelial cells were significantly lower than in group 1 (group 3, $\mathrm{P}<0.05$; group $4, \mathrm{P}<0.005$ ). Caspase-3positive indices were significantly higher in groups 3 and 4 than in group 1 (group 3, $\mathrm{P}<0.01$; group $4, \mathrm{P}<0.001$ ). These results suggest that dietary cGSL had a potent chemopreventive effect in the present short-term colon carcinogenesis bioassays, and that this effect may be associated with the inhibition of ACF and MDF and the induction of apoptosis.
\end{abstract}

Correspondence to: Dr Nao Sunagawa, Tumor Pathology, University of the Ryukyus Faculty of Medicine, 207 Uehara, Nishihara-cho, Okinawa 903-0215, Japan

E-mail: k058710@eve.u-ryukyu.ac.jp

Key words: glycosphingolipid, rice bran, prevention, preneoplastic lesions, colon

\section{Introduction}

Sphingolipids are a group of structural and bioactive derivatives that have a long chain backbone called a sphingoid base (sphingosine). These compounds have been regarded as inert structural components of cell membranes. However, in recent years sphingolipids have emerged as an important group of signaling molecules involved in cellular events such as immunoresponse, differentiation, cell proliferation and apoptosis. It was demonstrated that ceramide causes growth inhibition and induces morphological differentiation in HL-60 human myelocytic leukemia and neuroblastoma cell lines $(1,2)$. Obeid et al reported that tumor necrosis factor- $\alpha$ stimulates the production of ceramide and the induction of apoptosis by the hydrolysis of sphingomyelin (3). Other reports have indicated that the proapoptotic effect of ceramide is induced by the activation of Fas or radiation $(4,5)$. As a result, sphingolipids are attracting attention as a promising target of cancer chemoprevention and therapy.

Aberrant crypt foci (ACF) were proposed as colon preneoplastic lesions by Bird $(6,7)$ and are widely used as reliable biomarkers of colon carcinogenesis (8). Mucin-depleted foci (MDF) are lesions observed as mucin defects in rat colonic mucosa stained with high iron diamin-Alcian blue, and their histological characteristics demonstrate dysplastic features (9). Additionally, MDF are significantly correlated with the development of colon tumors in azoxymethane (AOM)- or 1,2-dimethylhydrazine (DMH)-treated rats (9-11). Furthermore, both ACF and MDF are valuable biomarkers for short-term colon carcinogenesis bioassays, and are useful for the examination of possible chemopreventive effects of a wide variety of candidate agents $(12,13)$.

Complex sphingolipids such as glycosphingolipid and sphingomyelin display biological activities similar to those of ceramide. Recent studies have shown that dietary intake of synthesized or naturally-occurring sphingomyelin and glycosphingolipids prevents colon carcinogenesis in DMH-treated or multiple intestinal polyposis (Min) mice (14-18).

Sphingolipids can be found in milk, eggs and soybeans; foods that are commonly consumed in everyday life as a dietary supplement. Although sphingolipids exhibit a variety 
of biological activities, the precise mechanisms by which the complex causes tumor suppression are not known.

In the present study, we examined whether crude glycosphingolipid (cGSL) obtained from rice bran actually inhibits the development of carcinogen-induced preneoplastic lesions such as ACF and MDF in the rat colon. Furthermore, to investigate its mechanisms of action on cell proliferation and apoptosis, immunohistochemical analysis of proliferating nuclear cell antigen (PCNA) and cleaved caspase-3 was performed.

\section{Materials and methods}

Animals, diets and chemicals. Four-week-old male F344 rats were purchased from Japan SLC Inc. (Hamamatsu, Japan). All animals were housed in wire cages (3 rats/cage) with free access to drinking water and the control diet (CE-2, Clea Japan Inc., Tokyo, Japan) under controlled humidity $(50 \pm 10 \%)$, lighting (12-h light/dark cycle) and temperature $\left(23 \pm 2^{\circ} \mathrm{C}\right)$ conditions. Composition of the CE-2 diet was water $(8.6 \%)$, protein $(24.9 \%)$, fat $(4.6 \%)$, fiber $(3.7 \%)$, ash $(6.7 \%)$ and nitrogen-free extract $(51.4 \%)$. cGSL was provided by Oryza Oil \& Chemical Co. Ltd. (Owari-Ichinomiya, Japan), and was extracted from rice bran by the ethanol extraction method. High performance liquid chromatography (HPLC) analysis revealed that the cGSL contained monoglucosylceramide (8.6\%), sterol (5.0\%), triglyceride and diglyceride (26.4\%), calcium carbonate $(53.0 \%)$ and starch $(7.0 \%)$.

Experimental design. Animal experiments were approved by the Animal Welfare Committee of the University of the Ryukyus. After being quarantined for 1 week, 42 male F344 rats were divided into 6 groups. Starting at 5 weeks of age, the rats in groups 1 to 4 ( $n=9 /$ group) received AOM (Nard Institute Ltd., Hyogo, Japan, $20 \mathrm{mg} / \mathrm{kg}$ body weight, s.c. injection) once a week for 2 weeks. Rats in groups 2,3 and 4 were fed a diet containing 250, 1,000 and 3,000 ppm cGSL, respectively, throughout the experiment. The concentration of cGSL was determined according to our previous study (19). Rats in group $5(n=3)$ were fed a diet containing 3,000 ppm cGSL to examine the adverse side effects of the complex. Rats in group $6(n=3)$ were fed the control diet alone and served as negative controls. At 5 weeks from the start date, all animals were euthanized under $\mathrm{CO}_{2}$ anesthesia. Colon tissues were removed, washed with saline, opened longitudinally and fixed with $10 \%$ buffered formalin.

Detection of aberrant crypt and mucin-depleted foci. The staining procedure for ACF and MDF was performed as described in our previous study (11). The fixed colon tissues were stained in an Alcian blue solution (Sigma Chemical Co., St. Louis, MO) for $5 \mathrm{~min}$ and immediately washed with distilled water, then placed on a glass plate with the mucosal side up. Using a light microscope at a magnification of $\mathrm{x} 40$, $\mathrm{ACF}$ and MDF were counted. Briefly, ACF were identified according to the following criteria: larger than and elevated above the adjacent normal crypts, with thickened cell walls lining the crypt and increased pericryptal area (11). MDF were identified as focal lesions characterized by the absence or very small production of mucins (11).
Immunohistochemical analysis. After ACF and MDF counting, colon tissues were rolled up, embedded in a Swiss roll form and subjected to immunohistochemical staining of PCNA and cleaved caspase-3. Immunohistochemical staining of PCNA is widely applied to examine cell proliferation (20). Caspase-3 is a pivotal apoptotic protein. Its active form, cleaved caspase- 3 , is generated by the proteolysis of its zymogen and exists only in the activated apoptotic cascade. Therefore, the detection of cleaved caspase- 3 is a sensitive indicator of apoptosis (21). Sections (4- $\mu \mathrm{m})$ were deparaffinized and rehydrated, then incubated in $3 \% \mathrm{H}_{2} \mathrm{O}_{2}$ for $20 \mathrm{~min}$ in order to block endogenous peroxidase activity. Sections were boiled in $1 \mathrm{mM}$ EDTA (pH 8.0) for $10 \mathrm{~min}$ in a pressure cooker. Subsequently, the sections were incubated with primary antibodies of PCNA (1:100 dilution, Dako Co. Ltd., Kyoto, Japan) or cleaved caspase-3 (1:200 dilution, Cell Signaling Technology Inc., Beverly, MA) at room temperature for $60 \mathrm{~min}$, then secondary antibody treatment was performed using Histofine Simple Stain (Nichirei, Tokyo, Japan). Visualization of immunoreactivity was performed with the Liquid DAB Substrate Chromogen System (Dako Co., Copenhagen, Denmark). To determine the PCNA-positive (PCNA PI) and caspase-3positive indices (caspase-3 PI), 15 visible crypts per colon were chosen, and at least 1,500 epithelial cells were counted. Positive indices were calculated as the percentage of positive cells with respect to the total number of cells counted.

Statistical analysis. One-way ANOVA was performed to analyze the data, and statistical differences were determined by Dunnet's test. All statements of significance are $\mathrm{P}<0.05$.

\section{Results}

General observations. A total of 42 rats survived to the end of the experiment, and none developed colon tumors. To ascertain whether the dietary administrtion of cGSL caused any toxic adverse side effects on body weight gain, the rats were monitored on a weekly basis. AOM and/or cGSL were not observed to have any significant effects on body, liver or kidney weight (data not shown). Dietary cGSL at 250, 1,000 and 3,000 ppm caused no symptomatic side effects in any of the rats.

Inhibition by cGSL of the occurrence of aberrant crypt and mucin-depleted foci. All rats in groups 1-4 developed ACF and MDF in their colonic mucosa. No ACF or MDF were observed in any of the rats in groups 5 and 6 . The total number of ACF was significantly lower in AOM-treated rats fed a 1,000 and $3,000 \mathrm{ppm}$ cGSL diet than in the control rats $(1,000 \mathrm{ppm}$, $\mathrm{P}<0.01 ; 3,000 \mathrm{ppm}, \mathrm{P}<0.05$; Table I), and the total number of MDF was significantly lower in AOM-treated rats fed a cGSL diet than in the control rats $(250 \mathrm{ppm}, \mathrm{P}<0.001 ; 1,000 \mathrm{ppm}$, $\mathrm{P}<0.001 ; 3,000 \mathrm{ppm}, \mathrm{P}<0.05$; Table I). The number of ACF or MDF that contained $>4$ crypts was not modified by the treatments. These results indicate that dietary cGSL inhibits the occurrence of ACF and MDF induced by AOM.

Inhibition of proliferating cell nuclear antigen-positive index by cGSL on the colonic epithelial cells. To determine whether cGSL affects cell proliferation in carcinogen-treated colonic 
Table I. Inhibition of the occurrence of aberrant crypt and mucin-depleted foci by crude glycosphingolipid.

\begin{tabular}{llcccc}
\hline Group no. & \multicolumn{1}{c}{ Treatment } & Total no. of ACF & $\begin{array}{c}\text { No. of ACF } \\
\text { containing }>4 \text { ACs }\end{array}$ & $\begin{array}{c}\text { Total no. of MDF } \\
\text { No. of MDF } \\
\text { containing }>4 \text { ACs }\end{array}$ \\
\hline 1 & AOM & $138.2 \pm 24.8^{\mathrm{a}}$ & $13.8 \pm 4.9$ & $13.9 \pm 4.1$ & $0.6 \pm 0.7$ \\
2 & AOM+250 ppm cGSL & $116.1 \pm 16.8$ & $13.4 \pm 4.3$ & $8.2 \pm 1.9^{\mathrm{b}}$ & $0.3 \pm 0.5$ \\
3 & AOM+1,000 ppm cGSL & $97.9 \pm 17.7^{\mathrm{c}}$ & $10.7 \pm 4.3$ & $7.8 \pm 2.5^{\mathrm{b}}$ & $0.4 \pm 0.5$ \\
4 & AOM+3,000 ppm cGSL & $105.9 \pm 37.6^{\mathrm{d}}$ & $10.8 \pm 5.0$ & $9.8 \pm 2.4^{\mathrm{d}}$ & $0.3 \pm 0.7$ \\
5 & 3,000 ppm cGSL & 0 & 0 & 0 & 0 \\
6 & None & 0 & 0 & 0 & 0 \\
\hline
\end{tabular}

${ }^{\mathrm{a}}$ Mean \pm SD. Significantly different from group 1 : ${ }^{\mathrm{b}} \mathrm{P}<0.001,{ }^{\mathrm{c}} \mathrm{P}<0.01,{ }^{\mathrm{d}} \mathrm{P}<0.05$; Dunnet's test. cGSL, crude glycosphingolipid; ACF, aberrant crypt foci; MDF, mucin-depleted foci; ACs, aberrant crypts; AOM, azoxymethane.

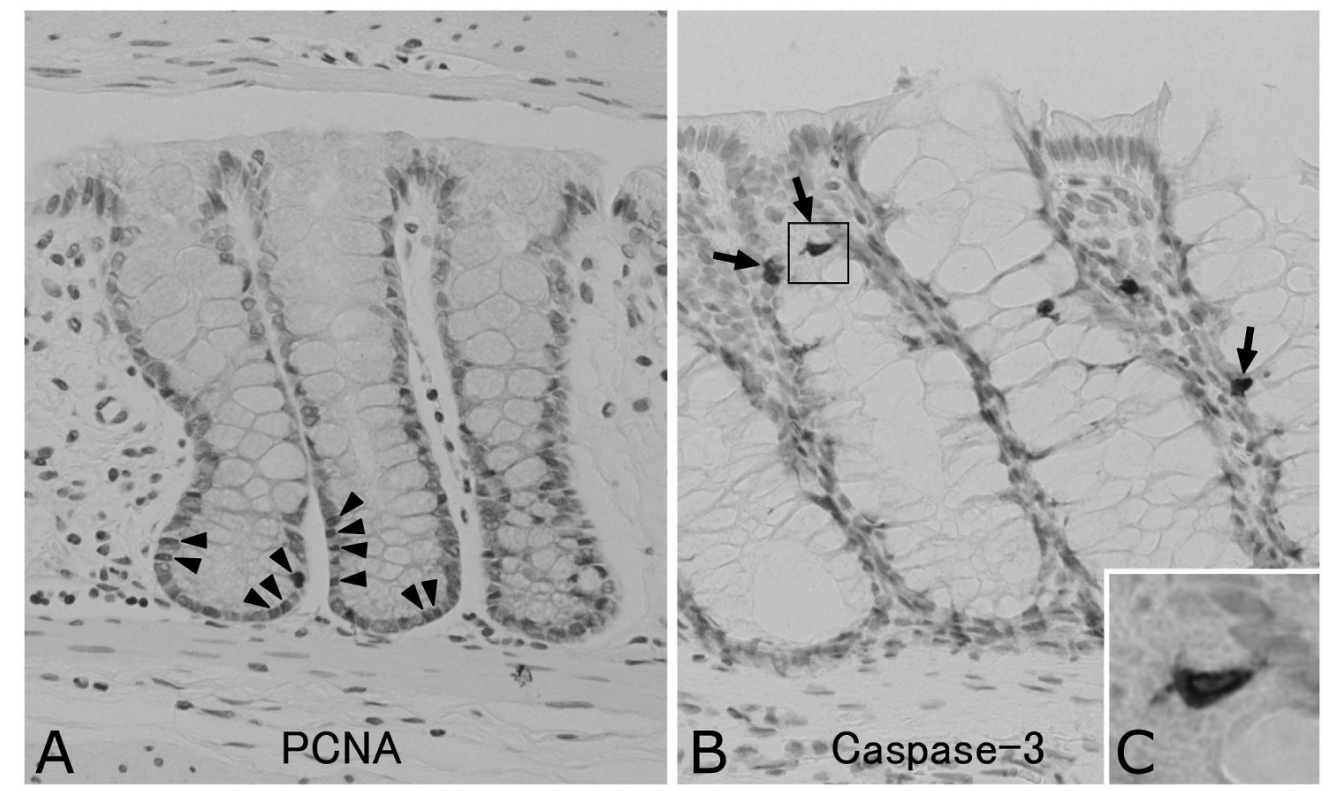

Figure 1. Representative results of immunohistochemical staining of PCNA (A) and caspase-3 (B and C) in the rat colonic epithelium. PCNA immunoreactivity is positive in the nucleus (arrow heads). Caspase-3-positive cells are mainly localized in the upper half of the crypt (arrows), and immunoreactivity shows the cytoplasmic pattern at high power magnification (C).

Table II. Inhibition of the proliferating nuclear cell antigenpositive index by crude glycosphingolipid in colonic epithelial cells.

\begin{tabular}{lll}
\hline Group no. & \multicolumn{1}{c}{ Treatment } & PCNA PI \\
\hline 1 & AOM & $5.28 \pm 1.18^{\mathrm{a}}$ \\
2 & AOM+250 ppm cGSL & $3.99 \pm 0.74$ \\
3 & AOM+1,000 ppm cGSL & $3.92 \pm 0.35^{\mathrm{b}}$ \\
4 & AOM+3,000 ppm cGSL & $3.47 \pm 0.42^{\mathrm{c}}$ \\
5 & 3,000 ppm cGSL & $3.29 \pm 0.50$ \\
6 & None & $3.25 \pm 0.34$ \\
\hline
\end{tabular}

${ }^{\mathrm{a}}$ Mean \pm SD. Significantly different from group 1 : ${ }^{\mathrm{b}} \mathrm{P}<0.05,{ }^{\mathrm{c}} \mathrm{P}<0.005$; Dunnet's test. PCNA PI, proliferating nuclear cell antigen-positive index; cGSL, crude glycosphingolipid; AOM, azoxymethane. mucosa, we measured the PCNA PI in epithelial cells by immunohistochemistry (Fig. 1). As shown in Table II, treatment of rats with 1,000 and 3,000 ppm cGSL caused a significant decrease in the PCNA PI $(1,000 \mathrm{ppm}, \mathrm{P}<0.05$; $3,000 \mathrm{ppm}, \mathrm{P}<0.005)$ as compared to the control group (group 1). This inhibition occurred in a dose-dependent manner. The PCNA PIs of groups 1 and 6 were 5.28 and 3.25, respectively. In groups 2, 3 and 4, the PCNA PI was 3.99, 3.92 and 3.47 in AOM-treated rats fed 250, 1,000 and 3,000 ppm cGSL, respectively. These findings suggest that dietary cGSL normalizes the increase in cell proliferation induced by AOM.

Effect of cGSL on apoptosis. Since cGSL is able to normalize increases in cell proliferation in carcinogen-treated colonic epithelial cells, we examined whether this inhibition by cGSL is due to the induction of apoptosis. After the injection of AOM, treatment of rats with 1,000 and 3,000 ppm cGSL 
Table III. Inhibition of the caspase-3-positive index by crude glycosphingolipid in colonic epithelial cells.

\begin{tabular}{lll}
\hline Group no. & \multicolumn{1}{c}{ Treatment } & Caspase-3 PI \\
\hline 1 & AOM & $0.55 \pm 0.67^{\mathrm{a}}$ \\
2 & $\mathrm{AOM}+250 \mathrm{ppm}$ cGSL & $0.62 \pm 0.16$ \\
3 & $\mathrm{AOM}+1,000 \mathrm{ppm}$ cGSL & $0.85 \pm 0.23^{\mathrm{b}}$ \\
4 & $\mathrm{AOM}+3,000 \mathrm{ppm}$ cGSL & $0.94 \pm 0.20^{\mathrm{c}}$ \\
5 & $3,000 \mathrm{ppm}$ cGSL & $1.01 \pm 0.13$ \\
6 & None & $0.97 \pm 0.04$ \\
\hline
\end{tabular}

${ }^{\mathrm{a}}$ Mean $\pm \mathrm{SD}$. Significantly different from group 1 : ${ }^{\mathrm{b}} \mathrm{P}<0.01,{ }^{\mathrm{c}} \mathrm{P}<0.001$; Dunnet's test. PI, positive index; cGSL, crude glycosphingolipid; AOM, azoxymethane.

caused a significant increase in the caspase- 3 PI as compared to the control group (group 1) $(1,000 \mathrm{ppm}, \mathrm{P}<0.01 ; 3,000 \mathrm{ppm}$, $\mathrm{P}<0.001$; Table III). This increase occurred in a dosedependent manner. These results indicate that cGSL induces apoptosis in colonic epithelial cells treated with AOM.

\section{Discussion}

Complex sphingolipids display a wide spectrum of biological activities including cell proliferation, differentiation and apoptosis. However, the precise mechanisms by which this complex exerts a cancer preventive effect are unknown. This study provides the first detailed examination of the inhibitory effects of rice bran-derived cGSL, which was extracted with safe solvents only, on 2 different categories of carcinogeninduced rat colon preneoplastic lesions, ACF and MDF. In the present study, we found that dietary administration of cGSL significantly inhibited the occurrence of ACF and MDF induced by the carcinogen AOM. We also found that the PCNA PI in colonic epithelial cells was inhibited in a dose-dependent manner by the treatment of rats with cGSL. Furthermore, dietary cGSL increased the caspase-3 PI in AOM-treated colonic epithelial cells. These findings suggest that cGSL prevents colon preneoplastic lesions, and that this effect may be associated with the inhibition of cell proliferation and the induction of apoptosis. Similar results have been reported in previous studies, which demonstrated that dietary intake of synthesized ceramide and milk-derived sphingolipids inhibited colonic cell proliferation in DMHtreated or Min mice $(18,22)$. As well, sphingosine, sphinganine and $\mathrm{C}_{2}$-ceramide induced apoptosis in HT29 and HCT116 human colon carcinoma cell lines (23). These findings, together with the results of the current study, suggest that cGSL has cancer preventive potential in a short-term colon carcinogenesis bioassay.

In the present study, the suppressive effect of cGSL on the occurrence of ACF and MDF was not statistically significant at a lower dose (250 ppm). At higher doses $(1,000$ and 3,000 $\mathrm{ppm}$ ), the inhibitory effect of cGSL on the occurrence of ACF and MDF was not dose-dependent. It is possible that a dose level of 250 ppm cGSL was not sufficient to suppress the formation of these lesions, while at $1,000 \mathrm{ppm}$ cGSL had already reached its maximum effect. It seems likely that had we used a larger number of animals, and had the experiment continued for a longer period, the number of ACF or MDF would have decreased further in the high-dose cGSL-treated groups.

Ceramide, sphingosine and sphinganine are considered to play a pivotal role in tumor suppression $(24,25)$. Schmelz et al have suggested that complex sphingolipids in food are hydrolyzed to ceramide in the intestinal tract and taken up by colonic cells, thus performing its biological activity $(16,17)$. Most sphingolipids are hydrophobic, and are thus insoluble in aqueous solution. There have been no studies investigating the colon cancer preventive effect of cGSL, and no epidemiological data are available regarding the relation between the daily consumption of sphingoid-rich food (e.g., rice bran, milk, eggs and soybeans) and colon cancer risk. Therefore, to clarify whether its effect is confined to colon cancer requires further investigation. The ceramide-mediated signaling pathways involved in tumor suppression are extensive $(24,25)$. The induction of apoptosis and the inhibition of cell proliferation are considered to be key events in tumor suppression $(23,26)$. Molecules such as RB, Bcl-2, p53, mitogen-activated protein kinases and protein kinase $\mathrm{C}$ are the downstream targets of ceramide $(24,25)$. Ceramide activates $\mathrm{Bcl}-2$, and this proapoptotic protein eventually activates caspase family molecules through the release of cytochrome $\mathrm{c}$ from mitochondria $(27,28)$. Schmelz et al demonstrated that sphingolipids normalize the localization of the $\beta$-catenin protein in the colonic mucosa (29). Aberrant expression of the B-catenin protein has been demonstrated in carcinogen-induced MDF in rats $(10,11)$. We found that the development of MDF was inhibited by cGSL treatment, suggesting that the alteration of the signaling pathway via $\beta$-catenin may play an important role in the modulation of carcinogen-induced colon carcinogenesis. However, this aspect is in need of further study.

In summary, we demonstrated the inhibitory effect of dietary cGSL on 2 different biomarker lesions, ACF and $\mathrm{MDF}$, in azoxymethane-treated F344 rats. The conceivable mechanism of action of cGSL might be the inhibition of cell proliferation and the induction of apoptosis in the colonic mucosa. With these considerations, cGSL may have had a potent chemopreventive effect in our short-term colon carcinogenesis bioassay system. Further studies are in progress to determine whether dietary cGSL suppresses tumor formation in long-term experiments.

\section{Acknowledgements}

This work was supported, in part, by a Grant-in-Aid from the Ministry of Health, Labor and Welfare and a Grant-in-Aid from the Ministry of Education, Culture, Sports, Science and Technology of Japan. We thank Tomoko Sugishita and Koji Kanazawa (Oryza Oil \& Fat Chemical Co. Ltd.) for supplying cGSL.

\section{References}

1. Okazaki T, Bielawska A, Bell RM and Hannun YA: Role of ceramide as a lipid mediator of 1 alpha,25-dihydroxyvitamin D3-induced HL-60 cell differentiation. J Biol Chem 265: 15823-15831, 1990. 
2. Riboni L, Prinetti A, Bassi R, Caminiti A and Tettamanti G: A mediator role of ceramide in the regulation of neuroblastoma Neuro2a cell differentiation. J Biol Chem 270: 26868-26875 1995 .

3. Obeid LM, Linardic CM, Karolak LA and Hannun YA: Programmed cell death induced by ceramide. Science 259: 1769-1771, 1993

4. Cifone MG, De Maria R, Roncaioli P, et al: Apoptotic signaling through CD95 (Fas/Apo-1) activates an acidic sphingomyelinase. J Exp Med 180: 1547-1552, 1994.

5. Haimovitz-Friedman A, Kan CC, Ehleiter D, et al: Ionizing radiation acts on cellular membranes to generate ceramide and initiate apoptosis. J Exp Med 180: 525-535, 1994.

6. Bird RP: Observation and quantification of aberrant crypts in the murine colon treated with a colon carcinogen: preliminary findings. Cancer Lett 37: 147-151, 1987.

7. Bird RP: Role of aberrant crypt foci in understanding the pathogenesis of colon cancer. Cancer Lett 93: 55-71, 1995.

8. Corpet DE and Tache S: Most effective colon cancer chemopreventive agents in rats: a systematic review of aberrant crypt foci and tumor data, ranked by potency. Nutr Cancer 43: 1-21, 2002.

9. Caderni G, Femia AP, Giannini A, et al: Identification of mucindepleted foci in the unsectioned colon of azoxymethane-treated rats: correlation with carcinogenesis. Cancer Res 63: 2388-2392, 2003.

10. Femia AP, Bendinelli B, Giannini A, et al: Mucin-depleted foci have beta-catenin gene mutations, altered expression of its protein, and are dose- and time-dependent in the colon of 1,2-dimethylhydrazine-treated rats. Int J Cancer 116: 9-15, 2005

11. Yoshimi N, Morioka T, Kinjo T, et al: Histological and immunohistochemical observations of mucin-depleted foci (MDF) stained with Alcian blue, in rat colon carcinogenesis induced with 1,2-dimethylhydrazine dihydrochloride. Cancer Sci 95: 792-797, 2004

12. Femia AP, Dolara P and Caderni G: Mucin-depleted foci (MDF) in the colon of rats treated with azoxymethane (AOM) are useful biomarkers for colon carcinogenesis. Carcinogenesis 25: 277-281, 2004.

13. Pierre Freeman A, Tache S, van der Meer R and Corpet DE: Beef meat and blood sausage promote the formation of azoxymethane-induced mucin-depleted foci and aberrant crypt foci in rat colons. J Nutr 134: 2711-2716, 2004.

14. Dillehay DL, Webb SK, Schmelz EM and Merrill AH Jr: Dietary sphingomyelin inhibits 1,2-dimethylhydrazine-induced colon cancer in CF1 mice. J Nutr 124: 615-620, 1994.

15. Schmelz EM, Bushnev AS, Dillehay DL, Liotta DC and Merrill AH Jr: Suppression of aberrant colonic crypt foci by synthetic sphingomyelins with saturated or unsaturated sphingoid base backbones. Nutr Cancer 28: 81-85, 1997.
16. Schmelz EM, Dillehay, DL, Webb SK, Reiter A, Adams J and Merrill AH Jr: Sphingomyelin consumption suppresses aberrant colonic crypt foci and increases the proportion of adenomas versus adenocarcinomas in CF1 mice treated with 1,2-dimethylhydrazine: implications for dietary sphingolipids and colon carcinogenesis. Cancer Res 56: 4936-4941, 1996.

17. Schmelz EM, Sullards MC, Dillehay DL and Merrill AH Jr: Colonic cell proliferation and aberrant crypt foci formation are inhibited by dairy glycosphingolipids in 1,2-dimethylhydrazinetreated CF1 mice. J Nutr 130: 522-527, 2000.

18. Symolon H, Schmelz EM, Dillehay DL and Merrill AH Jr: Dietary soy sphingolipids suppress tumorigenesis and gene expression in 1,2-dimethylhydrazine-treated $\mathrm{CF} 1 \mathrm{mice}$ and $\mathrm{ApcMin} /{ }^{+}$mice. J Nutr 134: 1157-1161, 2004.

19. Inamine M, Suzui M, Morioka T, et al: Inhibitory effect of dietary monoglucosyl-ceramide 1-O-beta-glucosyl-N-2'-hydroxyarachidoyl-4,8-sphingadienine on two different categories of colon preneoplastic lesions induced by 1,2-dimethylhydrazine in F344 rats. Cancer Sci 96: 876-881, 2005

20. Hall PA and Woods AL: Immunohistochemical markers of cellular proliferation: achievements, problems and prospects. Cell Tissue Kinet 23: 505-522, 1990.

21. Gown AM and Willingham MC: Improved detection of apoptotic cells in archival paraffin sections: immunohistochemistry using antibodies to cleaved caspase 3. J Histochem Cytochem 50: 449-454, 2002

22. Schmelz EM, Bushnev AS, Dillehay DL, Sullards MC, Liotta DC and Merrill AH Jr: Ceramide-beta-D-glucuronide: synthesis, digestion, and suppression of early markers of colon carcinogenesis. Cancer Res 59: 5768-5772, 1999.

23. Ahn EH and Schroeder JJ: Sphingoid bases and ceramide induce apoptosis in HT-29 and HCT-116 human colon cancer cells. Exp Biol Med 227: 345-353, 2002.

24. Ogretmen B and Hannun YA: Biologically active sphingolipids in cancer pathogenesis and treatment. Nat Rev Cancer 4: 604-616, 2004.

25. Yang J, Yu Y, Sun S and Duerksen-Hughes PJ: Ceramide and other sphingolipids in cellular responses. Cell Biochem Biophys 40: 323-350, 2004

26. Kolesnick R: The therapeutic potential of modulating the ceramide/ sphingomyelin pathway. J Clin Invest 110: 3-8, 2002.

27. Heinrich M, Wickel M, Schneider-Brachert W, et al: Cathepsin D targeted by acid sphingomyelinase-derived ceramide. EMBO J 18: 5252-5263, 1999.

28. Selzner M, Bielawska A, Morse MA, et al: Induction of apoptotic cell death and prevention of tumor growth by ceramide analogues in metastatic human colon cancer. Cancer Res 61: 1233-1240, 2001.

29. Schmelz EM, Roberts PC, Kustin EM, et al: Modulation of intracellular beta-catenin localization and intestinal tumorigenesis in vivo and in vitro by sphingolipids. Cancer Res 61: 6723-6729, 2001. 
